This is a pre-publication draft. The final version of this paper is published in The Journal of Clinical Nursing, 24(11-12), 1718-1729. http://dx.doi.org/

10.1111/jocn.12779

Any citations should refer to that version

Involving patients in understanding hospital infection control using visual methods

Mary Wyer, Debra Jackson, Rick Iedema, Su-Yin Hor, Gwendolyn L Gilbert, Christine Jorm, Claire Hooker, Matthew Vincent Neil O'Sullivan and Katherine Carroll 


\title{
Involving patients in understanding hospital infection control using visual
}

\section{methods}

\begin{abstract}
Aim and Objectives: This paper explores patients' perspectives on infection prevention and control.
\end{abstract}

Background: Healthcare-associated infections are the most frequent adverse event experienced by patients. Reduction strategies have predominantly addressed frontline clinicians' practices; patients' roles have been less explored.

Design: Video-reflexive ethnography.

Methods: Fieldwork undertaken at a large metropolitan hospital in Australia involved 300 hours of ethnographic observations, including eleven hours of video footage. This paper focuses on eight occasions where video footage was shown back to patients in one-on-one reflexive sessions.

Findings: Viewing and discussing video footage of clinical care enabled patients to become articulate about infection risks, and to identify their own roles in reducing transmission. Barriers to detailed understandings of preventative practices and their roles included lack of conversation between patients and clinicians about infection prevention and control, and being ignored or contradicted when challenging perceived suboptimal practice. It became evident that to compensate for clinicians' lack of engagement around infection control, participants had developed a range of strategies, of variable effectiveness, to protect themselves and others. Finally, the reflexive process engendered closer scrutiny and a more critical attitude to infection control that increased patients' sense of agency.

Conclusion: This study found that patients actively contribute to their own safety. Their success, however, depends on the quality of patient-provider relationships and conversations. Rather than treating patients as passive recipients of infection control practices, clinicians can support and engage with patients' contributions towards achieving safer care.

Relevance to clinical practice: This study suggests that if clinicians seek to reduce infection rates they must start to consider patients as active contributors to infection control. Clinicians can engage patients in conversations about practices and pay attention to patient feedback about infection risk. This will broaden clinicians' understandings of infection control risks and behaviours and assist them to support appropriate patient self-care behaviour.

Keywords: infection prevention and control; video-reflexive ethnography; patient involvement; healthcare-associated infection; qualitative research, MRSA 
SUMMARY BOX

What does this paper contribute to the wider global clinical community?

- Infection prevention \& control, patient experience and patient empowerment are important and timely issues for healthcare professionals globally

- This paper examines rarely researched in situ practices in Australia, offering a fresh look at infection prevention and control from the patient's perspective

- An innovative research design that harnesses the expertise of frontline healthcare professionals, patients and families to improve patient safety. 


\section{Involving patients in understanding hospital infection control using visual methods}

\section{INTRODUCTION AND BACKGROUND}

Healthcare-associated infections (HAIs) are currently the number one adverse event experienced by patients worldwide (World Health Organisation [WHO] 2011), despite a good understanding of transmission mechanisms and teaching on preventative practices being provided in both healthcare education facilities and the workplace (Vincent 2006). Reports have estimated that between 30-70\% of HAIs are preventable (Umscheid et al. 2011, Vandijck et al. 2013), meaning that many patients are experiencing needless pain, prolonged hospital stays, financial burden and even death (WHO 2011). The increasing prevalence of infection due to hospitalacquired multi-drug resistant organisms (MDROs) has further increased the mortality and morbidity associated with infections (WHO 2012), making HAIs a significant and pressing patient safety issue.

To date, the bulk of patient safety literature has been devoted to establishing whether 'clinical practice complies with existing evidence and guidelines' (Iedema et al. 2013, p. 28). This can clearly be seen in regard to infection prevention and control (IPC) strategies where the primary focus is on surveillance and reporting of compliance with guidelines such as the Five Moments of Hand Hygiene (WHO 2009). Despite these guidelines being standardised in policy and procedure, infections 
continue to spread. Furthermore, there is evidence that healthcare professionals have highly variable understandings of, attitudes to, and compliance with IPC strategies (Pittet 2000, Rickard 2004).

Arguments have been made that IPC recommendations fail to attend to the complexity of care at the frontline, and that clinicians, researchers and policymakers must become more intelligent about reducing infection transmission by confronting the everyday complexity of IPC, including the roles that patients may play in identifying infection risk and preventing cross-infection (Hughes et al. 2011, Wyer et al. 2014). Wyer et al. (2014) cite research that empirically demonstrates patients' as active contributors to their own safety (Hor et al. 2013) and urge that this type of research be extended to consider patients' perspectives of and involvement in, moment-to-moment IPC practices. While Wyer et al. (2014) recognise that patient-centred hand hygiene programs (McGuckin \& Govednik 2013) are designed to encourage patient empowerment around IPC, such programs rely on strategies that require patients to 'check up' on clinicians' hand-washing practices. Therefore, considerable clinician buy-in must also be achieved to prevent tensions in the patient-provider relationship (Hrisos \& Thompson 2013). Additionally, such strategies often derive from clinician or researcher notions of patient roles in IPC, not from patients' viewpoints (Hor et al. 2013).

This paper explores patients' experiences, understandings and enactments of IPC by researching alongside, and with, patients during their experiences of care. We report on four study aims in this paper: 1) to understand the complexity of IPC 
practices as seen through patients' eyes; 2) to explore the challenges that patients may face in trying to understand IPC and in having their infection control needs met; 3) to explore the possibilities of patients' contributions to reducing HAIs; and finally, 4) by using an interventionist methodology, to support patients to come to better understandings of IPC practice and how they may better position themselves in these practices. Thus patients may become empowered to develop new strategies and positionings that may assist them to contribute more effectively to the infection control safety of their own care and that of others.

\section{METHODS}

\section{Design}

This study was nested within a larger multi-method 3-year project on infection control. The aim of the larger project was to strengthen clinicians' awareness of cross infection risk and was conducted in two metropolitan teaching hospitals in Sydney, Australia. The invitation to patients to become active research participants was designed as a sub-study by the $\mathrm{PhD}$ candidate (first author) associated with the project. The sub-study was conducted in conjunction with the larger project at one site only; a 66 bed, adult surgical unit. The findings of the sub-study are the focus of this paper.

Both the larger project and the sub-study employed an 'interventionist' ethnographic approach (Mesman 2007) known as video-reflexive ethnography 
(Iedema et al. 2013). This method involves videoing care interactions and then showing the footage back to those videoed in reflexive sessions. The method departs from prevailing top-down, knowledge-accumulation approaches to patient safety, emphasising instead the expertise and insight of the people who actually provide and receive care. Drawing on the theory that people engage in the world primarily through often unconscious habit (Cohen 2009), video is used to reveal these habits and stimulate 'bottom-up' practice improvement. Video-reflexive ethnography has been used successfully in healthcare settings for more than a decade to challenge taken-for-granted practices and inspire discussions about the problems and potentials embedded in those practices (Iedema et al. 2013).

\section{Data collection}

\section{Recruitment and initial videoing}

\section{Clinicians}

Clinicians involved in both the larger project and the sub-study were acquainted with the purpose, risks and benefits of the project through information sessions, handouts, wall posters and emails. They were assured of a continuous consent process, in which consent was initially sought in written format, and then verbally negotiated prior to and during filming, and before any sections of footage were shown to other parties including patients. Consent was also required to participate in reflexive sessions as these were also filmed. Participation was voluntary and 
clinicians were able to withdraw at any time. Of the clinicians who were approached to participate in the sub-study, none refused.

Patients

Patient participation in the sub-study was initiated during a point prevalence survey of methicillin-resistant Staphylococcus aureus (MRSA) colonisation in the study site. During this survey, all patients in this unit were approached to have their nose and perineum swabbed by an infection control practitioner (ICP). Prior to the survey, all patients in the unit were approached by the researcher (first author), informed about the study and asked if they would be willing to be involved in video-reflexive sessions around upcoming infection control activities. A participant information and consent form was discussed and left with the patient for consideration. Later in the day the researcher returned to answer any questions and to gauge patients' interest in participating. On the day of the point prevalence survey the researcher shadowed the ICPs, and asked previously identified patients, who had expressed interest, for consent to video the nasal swabbing process. 12 patients agreed to be videoed. A small hand-held camera was used for this purpose. At a later date two more patients became aware of the study and asked to be involved. Videoing of care interactions for these patients included: (i) filming activities in an isolation room for two and half hours, and (ii) filming of a vacuum dressing change.

As such, total of 14 participants - eight female and six male - were videoed. Eight patients subsequently took part in reflexive sessions, of whom six had experienced 
an HAI or colonisation; five due to MRSA, one patient was colonised with MRSA, but unaware of it at the time of data collection and two were not colonised or infected.

\section{Video-reflexive sessions}

Of the 12 patients videoed for the point prevalence survey, five were discharged before a reflexive session could take place and one patient was unwell and withdrew from the study. The remaining six agreed to view footage in an individual reflexive session with the researcher. Five of these sessions took place at the patient bedside on the day after the point prevalence survey. The sixth session took place later in a hospital tutorial room. Reflexive sessions for the two patients who joined the study at a later date occurred at the bedside on the same day as the filming.

Each reflexive session lasted approximately 20-30 minutes. The footage was downloaded to a laptop that was positioned so both patient and researcher could view it comfortably. The video was played repeatedly, first with sound and then without, to enable discussion. These sessions were not overly structured so as to encourage open dialogue. Drawing on the principle of 'exnovation' (Iedema et al. 2013, p. 10-12), this process aimed to engage participants not only with the professional activities of clinicians, but also with the patient's own behaviours during care interactions. Thus patients were asked to discuss their own behaviours, beliefs and strategies around infection control. Four patients consented to having the reflexive session videotaped, with both researcher and patient positioned in the frame. The other four patients agreed to have the session audiotaped. 


\section{Analysis}

When using video-reflexive methods the line between researcher and participants as data creators and analysts is intentionally blurred (Iedema et al. 2013). Videoreflexivity encourages co-interpretation of data between researchers and participants, as contributing to the rigour and accountability for the analysis (Carroll 2009). Data analysis therefore began when participants and researcher watched and discussed the footage together during reflexive sessions. Subsequently, these recorded discussions were transcribed and entered into Dedoose qualitative data analysis software. Themes and subthemes were identified by the researcher and again discussed with participants as well as the wider research team over a period of many months following the data collection. As a result these themes changed over time as they continued to be refined by all parties.

\section{Ethical considerations}

This method of enquiry and the use of camera and video footage raises special issues with regard to the power relations between researcher and patients. To address these issues this study was guided by principles drawn from feminist approaches (Kindon 2003, Carroll 2009) and visual ethics frameworks (Papademas 2009). Informed consent was obtained and participants were informed of the right to withdraw at any time. Each patient chose his or her own pseudonym, or in some cases asked to have their actual name used. Participants featured in vignettes in this paper gave explicit consent for photo use. These are pixelated to maintain their 
privacy. Non-pixelated images are of the first author (MW). This study received approval from the human research ethics committees the University of Technology, Sydney and the Western Sydney Local Health District.

\section{Findings}

The findings are presented under themes and subthemes (Table 1).

\section{Seeing IPC through patients' eyes}

\section{Patient experiences and understandings of IPC}

Viewing and discussing video footage of clinical care enabled patients to become increasingly articulate about infection control risks and practices. They were also able identify their own roles in reducing transmission.

Prior to admission, most patients had heard of HAI and MRSA and were aware that becoming infected could be life or limb threatening. However, most were not aware of the extent to which MRSA was present in hospitals and they had not given much thought to the personal risk of acquiring an MRSA during their hospital stay.

Sombie: [Large tertiary hospital] copped it a few years ago. Golden Staph...

R: So do you think [they] would still have it?

S: No

It was usually after patients had become colonised or infected that they developed heightened awareness of HAI and IPC and began to comprehend MRSA prevalence: 
There's a lot more MRSA patients than just me. Which you just learn that over time.

From different signs on the door ... and hearing nurses ... "Oh no he's MRSA." Or you see the people following protocol with gloves gown and everything and you're like, "Oh, MRSA too". (Rob)

Most patients were able to identify contact transmission risks. However, some were either unsure how MRSA transmission occurred, or held inaccurate beliefs:

I mean it can spread - like if I've got an infection and ... nurses are handling me and then they go to the next patient ... whatever the infection is, it's going to keep going around in the hospital. (Fiona)

From dirty water in the towers. Through the air conditioning system. (Sombie)

However, all participants were aware that rules were in place to guide practice and to help reduce infection transmission. Patients considered consistent use of personal protective equipment (PPE), cleaning, surveillance activities such as the point prevalence survey, and aseptic technique for invasive procedures as important to IPC. They also recognised the need for source isolation and treatment for infections. Despite the promotion of the Five Moments of Hand Hygiene with prominent signage displayed around the ward, the patients in this study placed greater emphasis on the use of gloves over hand hygiene as an important infection control measure. Discussions while watching the footage ultimately revealed, that for many patients, gloves had come to represent safety. For half of participants included in the point prevalence survey the first thing they noticed was the presence or absence of gloves: 
The first thing I noticed was she wasn't wearing gloves. Is that normal procedure? (Destiny)

In fact gloves were not required by hospital protocol and the ICPs performed appropriate hand hygiene throughout the point prevalence survey. However, most patients believed that gloves were important for preventing infection transmission. Some participants believed that gloves used in the footage were sterile when in fact they were just clean gloves:

She's already sterile...she's gloved. (James)

It was not specifically recognised by any participant that hand hygiene should be performed before donning gloves and initially only two patients believed that hand hygiene was just as good an option as gloves:

If they washed their hands in front of you or I seen them wash them at the tap and they didn't touch the tap afterwards that would be fine. (June)

I've had ... the odd doctor who's put in a cannula and have been un-gloved so they can feel it ... well their hands were washed so I felt no problems with contamination. (James)

Two patients initially stated that they had no role to play in IPC:

You don't think about that do you?...I mean you come in here, you do what they tell you to do and that's it. (Ann) 
Me? I can't really do much. (Fiona)

However, during video-reflexive discussion it became clear that all participants actively practiced IPC. They discussed activities such as washing their hands regularly, maintaining personal hygiene, agreeing to participate in surveillance activities such as the point prevalence survey, complying with treatment, speaking up when witnessing substandard IPC practices, following rules when instructed and keeping their bed-space clean and uncluttered. One participant clearly positioned patients as equal partners in preventing infection transmission:

We are like one big community. And everybody does a bit and it helps. (Sombie)

\section{Challenges to understanding IPC}

Participants expressed interest in IPC activities but described the difficulty they had in gaining information. The reflexive process enabled both the researcher and patients to understand just how important relationships and conversations were to developing an understanding of IPC and to patients' abilities to contribute effectively to their own safety and to the safety of others.

Lack of information exchange between patients and clinicians was evident. On admission none of the patients had received any information about HAI. If they acquired an MDRO the ICP would attempt to visit the patient with information but for a variety of reasons this did not always occur. Ann and James had both acquired 
MRSA on previous admissions. They recalled how they first learned of their positive status.

Actually they never told me before that I had Staph I just overheard them talking. (Ann)

...the first time that I learned about MRSA was during a pre-operation visit... I was wondering why they were all dressed in their [PPE]. And they were cleaning everything every time I touched something... And that's when ... the doctor told me I had MRSA. (James)

Fiona's wound swab showed positive for MRSA three days prior to the point prevalence survey but she was not yet aware of her status. This was despite the introduction of PPE and contact precaution signage above her door on the first day she was diagnosed.

MRSA had contributed to Rob's foot being amputated. He didn't understand why he was still in an isolation room:

... is it gone, because [my leg has] been discarded?...Do I still have it? If not, why am I still in this [isolation] ward?

This response shows a lack of understanding about the difference between colonisation and infection. As a result, Rob had been engaging in activities that may have contributed to the environmental contamination that was a problem in the unit at that time. He discussed the incongruity of staff efforts to don PPE when he could leave isolation and use the ward's shared facilities any time. He wondered whether 
he was spreading MRSA by doing this. When asked if he had ever questioned staff about this he responded:

B: No. There's nobody to ask ... I don't think they know enough about it.

R: What makes you think that?

B: ... well they don't seem to talk about it

In contrast, when viewing footage of her dressing, June noticed that it was easy to converse with the nurse; to question and make requests about how the dressing should be done. She recognised that it may not have been so easy with other nurses. When asked how important these conversations were she stated:

Very, very important. ...It makes me feel more at ease. They're doing it right and I'm not going to have to come back to this fucking place again [laughs].

All patients noticed inconsistencies in clinician adherence to IPC practices. The discussion above on gloves use is one example. The variable use of PPE when clinicians entered isolation rooms was also confusing for participants. Inconsistencies in staff practice meant that patients were not always sure of correct procedure. However not everyone questioned these inconsistencies as they believed the staff knew what they were doing:

I just thought, "She knows what she's doing. And it must be OK" (Destiny) 
Although most participants wanted to, and sometimes did, speak up when they perceived unsafe practice they did not always feel able to do so. Reasons for not speaking up included fear of offending clinicians and possible repercussions for future care. They also felt that attempts to engage with clinicians to learn more about IPC would result in them feeling negated or even berated. ... it would be hard to ask because they'd think you were undermining them. (Destiny)

If I offend them today, what are they going to do to me tomorrow? ... I find that because I'm not a professional, at times that talking to anyone on a professional subject I would at times get a response of, "Oh well I'm the professional and I can tell you that the likelihood of you catching something or anything like that is very low. " So I suppose a bit of that comes into it (James)

She could have snapped at me or something. "I know what I'm doing. You don't need to...I'm not touching your skin." (Fiona)

\section{Compensating for challenges}

To compensate for these challenges participants had developed a range of diversionary tactics to learn more about HAI and IPC. One patient had sought information about MRSA from the Internet. Some discussed IPC with other patients. Rob found it helpful to listen to nurses educate each other. 
Most patients observed staff practice and formed ideas about IPC from what they saw. Scrutinising staff practice shaped the patients' activities.

Well just by her behaviour, that she acted in the room. She was very like, strict. That's what made me sort of more strict. (Rob)

...like I said cause I've watched them I know. (June)

Through these compensatory measures the participants had developed understandings of HAI and IPC that were not always fully informed and resulted in a vague worry for some although enabling a more strategic approach for others. For some, IPC was of lesser concern than their surgical recovery, but others were more vigilant and had developed strategies that enabled them to feel they were actively contributing to IPC and their own safety. These strategies varied in their effectiveness. A few used humour or asked naïve questions of staff to signal their concerns about practice. Others praised good practice to indicate care preferences. When they couldn't directly observe staff, patients used other senses. June, Destiny and Fiona listened for the sound of hand hygiene being performed on the other side of the curtained bed-space. Vignette 1 captures the sensory ways in which James assessed his wound care, and the strategies he developed to achieve safer care for himself and others. These strategies, while creative, could arguably also be counterproductive. 


\section{Seeing IPC in a new light}

The video-reflexive process engendered closer scrutiny and a more critical attitude to IPC that was empowering for participants. James expressed the dramatic impact of watching the footage:

Like as I said, I hate seeing myself on video but I think it's such a great tool...in the sense of like people just don't understand what they are doing. Or at least have no knowledge of it. It's good.

\section{Closer scrutiny - new understandings}

Video feedback rapidly enabled participants to detect previously unrecognised transmission risks and come to new understandings about IPC. Scrutinising his bedspace on the screen, Clyde noticed that his large array of personal belongings were collecting dust and making it harder for the cleaners to clean properly. He was also surprised by the following realisation:

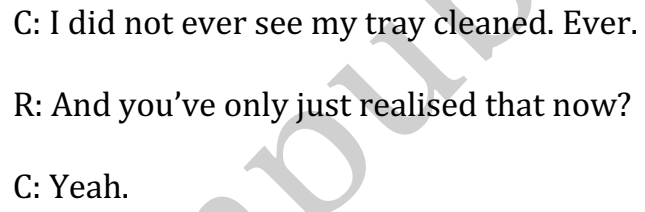

James observed one powerful example of the complexity of transmission risk. He noticed his phlebotomist had arrived already gloved from what he presumed was a clean field, but that she started touching things in the environment and contaminating her gloves before coming to take his blood: 
J: ...she's come from an outside source, she's gloved up ... That sink, everyman and his dog uses

it...like that light switch hasn't been touched this morning - but when was it last touched?

R: And then how does that equate? From her touching that stuff, to an issue for you with infection control?

J: She's drawing the blood. And needles and sharps. So it's not unlikely that there could be some contamination there.

R: Contamination going...?

J: Into me.

The reflexive process enabled some patients to notice and question their own habits. In particular they were alerted to how they positioned themselves in clinical interactions and how this affected their ability to have their IPC needs met. Rob had lost his leg partly due to HAI. Throughout the discussion he insisted he would speak up if clinicians did not don PPE when entering his room. However, he came to realise that he did not always speak up to doctors:

Because I'm thinking they should know ... they're in a highly trained position....and they should follow the rules too. More so than me. I'm just a patient sitting here... It's not me that has to follow the rules. Yet I'm the one sitting here with the disease ... it's another reason why I don't really bother to say anything to them. Because I think, "Oh well that's up to you. You should know." ... They're touching me and they're going to touch somebody else and they're going to touch somebody else. And there it goes through the hospital.

James also came to new understandings about risks and habits. Prior to watching the video he operated on the belief that phlebotomists used IPC practices primarily to protect themselves but that a follow-on effect of this would be that patients would 
also be protected. Seeing the footage he realised this was not the case but also recognised that he would probably not speak up about it:

Now seeing that... I honestly don't know if I'd pick them up on it. I honestly don't, I just really don't. I honestly don't know why... it's the fear of offending. Cause they're not conscious of what they're doing. They haven't gone out of their way to specifically try to injure you.

Vignette 2 describes how Clyde came to new understandings about IPC, moving from an assumption about the safety of gloves, to recognising that hand hygiene may be more beneficial for preventing transmission.

\section{A more critical view - new strategies}

These new insights and understandings increased patients' sense of agency. They were prompted to develop new strategies for IPC. Perhaps because of a fear of offending, some patients devised strategies that would allow clinicians to save face. In response to the scenario with the phlebotomist James decided:

... I'd probably try to grab her gloves or something - just destroy the field so she'd re glove...You know what I mean? Something stupid.

Destiny decided she would speak up if she noticed someone had not done hand hygiene but also made a back up plan:

I'd try. I don't know if I would do it every time. But I'd be wary of who and I'd tell my nurse. 
More aware of the lack of hand hygiene offered to bedbound patients, Ann came up with this solution, which she was able to achieve shortly after the reflexive session:

Yes I was thinking, I wonder why they don't give me like the antiseptic hand wash near my bed so I can use that more often. Like before I'm going to eat...

Clyde decided that more authority and direction from healthcare professionals was needed to reduce infection transmission:

People have to be told to do things if you're going to get the job done properly. [Pointing to hand gel in the video] You can't say, "Oh that's within reach of the patients, that's OK." It's not. One reason being that many patients are crook... and they're not focused on some of those things... So people need to come along and say, "Hey there mate - wash time." Even if it's every hour or whatever.

\section{DISCUSSION}

This study adopted an approach that embraced the everyday complexity of clinical practices and engaged with people at the frontline of care as a "critical source of insight and momentum for dealing with the rising levels of complexity of care" (Iedema et al. 2013, p. 1). We report hitherto unavailable findings about patient perspectives and enactments of infection control. Further, we show how patients, by viewing video footage of their own clinical care interactions, were given a new space in which to engage with practices that have the potential to affect their health. Through their eyes we have explored the complexity of in situ infection control 
practices and the ways in which rules and regulations play out variably at the front line of care.

Clinician adherence to hand hygiene is considered the leading preventative measure against HAI (Pittet et al. 2011) and correspondingly the bulk of the literature addressing patient roles in IPC focuses on patient-centered hand hygiene (Landers et al. 2012, McGuckin \& Govednik 2013). Through co-analysis of videoed in situ care interactions, this study has revealed numerous other participant-identified IPC practices and roles that patients can play to prevent transmission and to contribute to their own safety.

However, patients' experiences in this study corresponded with three significant barriers to patient involvement in safety as proposed by Howe (2006); intrapersonal, interpersonal and cultural (p. 528). Participants in the current study reported intrapersonal factors such as feeling physically and psychologically unable to focus on IPC because of more pressing concern about their underlying condition or surgery, as well as insufficient knowledge of IPC practices.

Interpersonal factors included communication deficits between healthcare workers and patients (Mutsonziwa \& Green 2012). Despite the push for patient engagement in infection control (WHO 2009, NHMRC 2010), at this study site at least, conversations between patients and clinicians about IPC practices and roles were noteworthy for their absence. Being largely unaware of the risks and preventative measures for HAI on admission meant that patients had limited capacity for 
contributing to IPC. Often it was a case of too little, too late once they acquired HAI, and even then they struggled to get the information they needed.

Patients also faced cultural marginalisation such as being ignored or challenged when speaking up about suboptimal practice or voicing care preferences. This resonates with findings from other research on patient experiences of IPC and HAIs (MacDonald 2008, Burnett et al. 2010, Dancer 2012). This apparent lack of clinician responsiveness towards patients' roles in infection control, denied the patients an opportunity to more effectively contribute to patient safety.

To compensate for this lack of engagement, patients observed and listened to clinicians as they performed IPC, and what they saw and heard shaped their attentions and precautions around infection risk. It is well documented, however, that clinicians do not always adhere to IPC rules (Pittet 2000, Rickard 2004) and all participants noticed inconsistent staff practices (Newton et al. 2001, Barratt et al. 2011). As a result, participants had developed skewed perceptions of IPC. Some experienced unnecessary anxiety about practice, especially around the use of gloves. Others held vague or erroneous notions about risk factors for HAI and the rationale behind IPC practices (Newton et al. 2001). From these limited understandings, a few patients had developed effective strategies to actively contribute to the safety of their care and the safety of others, but others had developed strategies that could be counterproductive. 
Finally, viewing and discussing video footage of clinical care also had a transformative impact on participants in this study. They came to more informed understandings about IPC and recognised disconnects between what they wanted from clinical interactions and how they behaved to get what they wanted. As with other research using video-reflexive methods (Iedema \& Rhodes 2010, Iedema et al. 2013), becoming more aware of their own behaviours compelled participants to revise their current responses to these disconnects and to develop new strategies for having their infection control needs met.

\section{CONCLUSION}

Despite the push in policy for patient engagement in quality and safety improvement, we know very little about patients' contributions to IPC. Previous research and commentary on patient involvement in IPC has tended to comprise clinician or researcher viewpoints on what patients might do to ensure safety or suggestions that patients should not be responsible for their own safety (Hill 2011, Wyer et al. 2014). The findings of this study, however, verifies arguments by Hor et al. (2013) that it is insufficient to discuss whether patients should be involved in ensuring their own safety - we now know that they already are involved. Their success, however, depends on the quality of patient-provider relationships and conversations. This paper has shown that accepting and engaging with patient involvement in IPC can be critical to safety and that ignoring patients' involvement can detract from safety. Furthermore, through the use of video methods that help to 
engage with complexity and encourage reflexivity, a broader understanding of the opportunities and potentials for patient involvement in IPC has been made possible.

HAIs are of critical global concern and current strategies are far from achieving optimal outcomes. We should be reminded that patients have the most at stake when it comes to the detrimental effects of infection, and rather than treating patients only as passive objects on which to perform IPC, clinicians can and should support and engage with patients' contributions to achieve safer patient care.

\section{Strengths and limitations}

A small sample size was one limitation of this study and participants were generally articulate and not extremely unwell at the time of the research. However, this is also a strength of the study in that the small sample size allowed for close and prolonged engagement with participants. Furthermore video-reflexivity offers more than a description of 'how things are'. The method also enables participants to realise 'what could be' and to become more active agents in infection control.

All participants were proficient English speakers; attempts to involve relatives of patients and/or patients with limited English were unsuccessful. Findings therefore may not be generalisable to minority patient populations who may face different challenges and opportunities. Another aspect of care-collaboration unexplored in this paper is the clinician's perspective. The video-reflexive method has also been employed to support patient-provider collaboration, and we will report clinician responses to this patient feedback in a separate paper. 


\section{RELEVANCE TO CLINICAL PRACTICE}

Findings of this study suggest that if clinicians seek to reduce HAI rates they must start to consider patients as active contributors to IPC. Clinicians should actively engage patients in conversations about IPC practices and pay attention to patient feedback about infection and infection risk. Such feedback may broaden clinicians' understandings of IPC risks and behaviours and can also assist clinicians to support appropriate patient self-care behaviour.

\section{CONFLICT OF INTEREST}

The authors declare that they have no conflicts of interest.

\section{Source of Funding}

This doctoral work was funded in the form of a research scholarship through a larger Australian government funded project: Strengthening clinicians' capacity for infection control: a multi-method study to reduce MRSA infection and transmission National Health and Medical Research Council 


\section{REFERENCES}

Barratt R, Shaban R \& Moyle W (2011) Behind barriers: Patients' perceptions of source isolation for methicillin-resistant Staphylococcus aureus (MRSA). Australian Journal of Advanced Nursing 28, 53-59.

Burnett E, Lee K, Rushmer R, Ellis M, Noble M \& Davey P (2010) Healthcareassociated infection and the patient experience: A qualitative study using patient interviews. Journal of Hospital Infection 74, 42-47.

Carroll KE (2009) Outsider, insider, alongsider: Examining reflexivity in hospitalbased video research. International Journal of Multiple Research Approaches 3, 246-263.

Cohen MD (2009) Reading Dewey: Some implications for the study of routine. In The Oxford Handbook of Sociology and Organization Studies (Adler P ed.), Oxford University Press, New York, pp. 444-463.

Dancer SJ (2012) Infection control 'undercover': A patient experience. Journal of Hospital Infection 80, 189-91.

Hill D (2011) Hand hygiene: Are we trying to make the patient the fail-safe system? Journal of Hospital Infection 79, 380-381.

Hor S, Godbold N, Collier A \& Iedema R (2013) Finding the patient in patient safety. Health: An Interdisciplinary Journal for the Social Study of Health, Illness and Medicine 17, 567-583.

Howe A (2006) Can the patient be on our team? An operational approach to patient involvement in interprofessional approaches to safe care. Journal of Interprofessional Care 20, 527-534.

Hrisos S \& Thomson R (2013) Seeing it from both sides: Do approaches to involving patients in improving their safety risk damaging the trust? An interview study. PLoS ONE 8, e80759.

Hughes J, Blackman H, McDonald E, Hull S \& Fitzpatrick B (2011) Involving service users in infection control practice. Nursing Times 107, 18-19. 
Iedema R, Mesman J \& Carroll K (2013) Visualising health care practice improvement: Innovation from within. Radcliffe Publishing, London.

Iedema R \& Rhodes C (2010) An ethics of mutual care in organizational surveillance. Organization Studies 31, 199-217.

Kindon S (2003) Participatory video in geographic research: A feminist practice of looking? Area 35, 142-153.

Landers T, Abusalem S, Coty MB \& Bingham J (2012) Patient-centered hand hygiene: The next step in infection prevention. American Journal of Infection Control 40, S11-7.

MacDonald P (2008) Exploring patients' experiences of MRSA to help reduce HCAIs. Nursing Times 104, 32-33.

McGuckin M \& Govednik J (2013) Patient empowerment and hand hygiene, 19972012. Journal of Hospital Infection 84, 191-199.

Mesman J (2007) Disturbing observations as a basis for collaborative research. Science As Culture 16, 281-295.

Mutsonziwa GA \& Green J (2012) Colonised and isolated: A qualitative metasynthesis of patients' experiences of being infected with multiple drug resistant organisms and subsequent isolation. Healthcare Infection $16,147-155$.

Newton JT, Constable D \& Senior V (2001) Patients' perceptions of methicillinresistant Staphylococcus aureus and source isolation: A qualitative analysis of source-isolated patients. Journal of Hospital Infection 48, 275280.

NHMRC (2010) Australian guidelines for the prevention and control of infection in healthcare. Commonwealth of Australia.

Papademas D (2009) IVSA code of research ethics and guidelines. Visual Studies 24, 250-257.

Pittet D (2000) Improving compliance with hand hygiene in hospitals. Infection Control and Hospital Epidemiology 21, 381-386.

Pittet D, Panesar SS, Wilson K, Longtin Y, Morris T, Allan V, Storr J, Cleary K \& Donaldson L (2011) Involving the patient to ask about hospital hand 
hygiene: A national patient safety agency feasibility study. Journal of Hospital Infection 77, 299-303.

Rickard NA (2004) Hand hygiene: Promoting compliance among nurses and health workers. British Journal of Nursing 13, 404-410.

Umscheid C, Mitchell M, Doshi J, Rajender A, Williams K \& Brennan P (2011) Estimating the proportion of healthcare-associated infections that are reasonably preventable and the related mortality and costs. Infection Control and Hospital Epidemiology 32, 101-114.

Vandijck D, Cleemput I, Hellings, J \& Vogelaers D (2013) Infection prevention and control strategies in the era of limited resources and quality improvement: A perspective paper. Australian Critical Care 26, 154-157.

Vincent C (2006) Patient safety. Elsevier Churchill Livingstone, Edinburgh.

World Health Organization (2009) WHO guidelines on hand hygiene in health care: First global patient safety challenge: Clean care is safer care. World Health Organization, Geneva.

World Health Organization (2011) Report on the burden of endemic health careassociated infection worldwide. World Health Organisation, Geneva.

World Health Organization (2012) The evolving threat of antimicrobial resistance: Options for action. World Health Organisation, Geneva.

Wyer M, Iedema R \& Hor S (2014) Editorial: Patients: Passive subjects or active participants in reducing the spread of infection? Journal of Clinical Nursing, 10.1111/jocn.12464 


\begin{tabular}{|l|l|l|}
\hline Themes & $\begin{array}{l}\text { Seeing IPC through } \\
\text { patients' eyes }\end{array}$ & Seeing IPC in a new \\
& light \\
\hline Subthemes & $\bullet$ Patient experiences and & $\bullet$ Closer scrutiny - new \\
understandings of IPC & understandings \\
& $\begin{array}{l}\bullet \text { Challenges to } \\
\text { understanding IPC }\end{array}$ & strategies \\
& challenges & \\
\hline
\end{tabular}

\section{Table 1: Themes and subthemes}




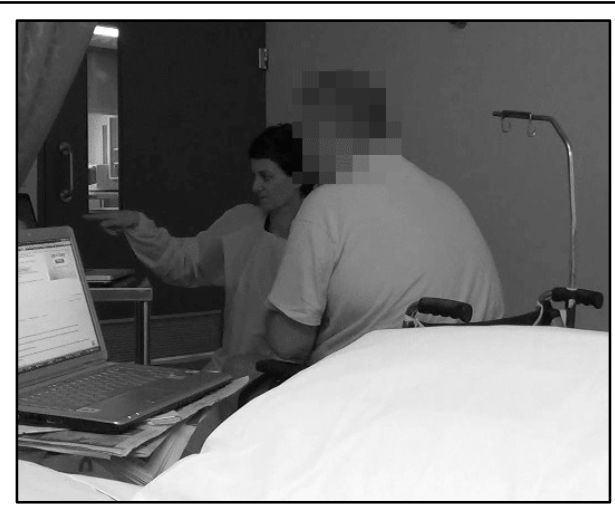

Vignette 1: Compensating for challenges

(These excerpts are taken from the original video-ethnographic data rather than reflexive data. First author is pictured, patient image is pixilated)

James was at risk of losing his leg due to a chronic diabetic foot ulcer that was infected with an MDRO. He had to lie prone for his dressing and couldn't see it being done. During a previous admission he had learned the correct technique for wound cleansing through listening to a clinical nurse consultant educating junior nurses. Since then, at each dressing change, he would sense how well the dressing was being done by feeling:

Wipe it one way, discard it.... can feel certain nurses using the one gauze in a lets clean the floor attitude' and other nurses doing the correct procedure.

When James felt unable to speak up about poor dressing technique he employed creative tactics to get better dressings:

As soon as I have a change of shifts, I'll remove the dressing myself and say it fell off and get it redressed.

James had never been informed of any precautions he should take as a patient isolated for MRSA. Through watching staff he had developed some understandings of contact precautions. He regularly left his room to use the communal kitchen but was anxious not to spread MRSA to others. He'd devised his own IPC strategy of using paper towels to touch equipment in the kitchen. 


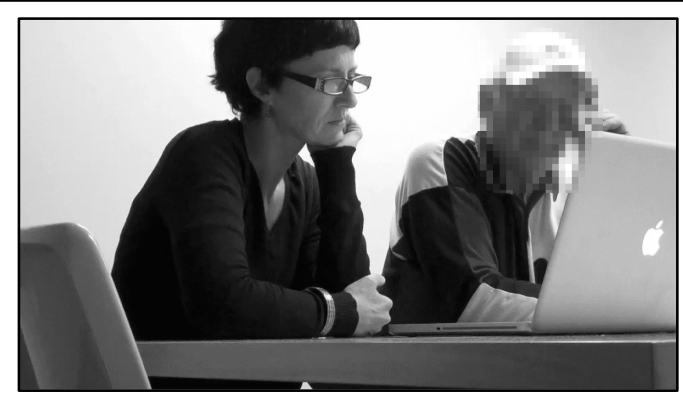

Vignette 2: Closer scrutiny - new understandings

(First author is pictured, patient image is pixilated)

Clyde watched footage of the ICP taking his nasal swab for the point prevalence survey. He initially believed that gloves should have been worn to prevent transmission of infection from the nurse to him. He had assumed that when clinicians use gloves during direct care they were sterile gloves. When he realised that for this procedure, had the nurse decided to wear gloves, she would have only donned clean gloves, he decided that hand-washing would be equally effective. Quickly though, he adjusted this line of thought again, considering that clean gloves possibly posed more of an infection risk for him than hand-washing alone.

So his thinking developed from feeling that care is safer through glove use to realising that not all gloves are safe.

C: She should wear gloves shouldn't she?

R: Why do you think she should wear gloves?

C: Well because she's approaching my person without gloves and that can transfer infection.

R: Transfer infection...?

C: To me

R: What if she has washed her hands beforehand? Would you still like her to have gloves on as well?

C: Yeah.

$\mathrm{R}:$ What do the gloves do that make you feel safer?

C: They're sterile. They're branded sterile aren't they? So anything that happens between putting them on and coming to me, it's a smaller risk.

$\mathrm{R}$ : The gloves that you see around that the nurses and doctors and whatnot put on. Do you see where they get them from? Or do they just arrive with gloves on?

C: No, no [stops to think]. They produce them. I don't know whether they bring them in their pocket or where. No they don't come with them. So they must have a stock somewhere.

R: Have you seen the ones that sit just outside the doorway in the boxes?

C: Those blue ones?

R: Yeah. They're the ones that they're putting on.

C: No - I've had the white ones.

R: The white ones they tend to use for dressings and they are sterile. But ... if this nurse was going to put gloves on she would just put blue ones on.

C: And what sort of sterility percentage are they? Are they sterile gloves or just gloves?

R: They're just clean gloves.

C: Well I'd be as happy with clean hands. It's just as good as the blue gloves. In fact they're probably worse, they're just hanging on the wall. Goodness knows what gets in there [laughs]. Correct? ... A glove to me is a sterile glove. It's not just a glove hanging off the wall. 